\title{
An unusual cause of trash feet
}

\author{
A $M$ Khan, S Jacobs
}

Answers on $p 794$.

A 65 year old man presented to the accident and emergency (A\&E) department with complaint of patchy mottling of both his feet. The following day this progressed to painful blisters and then to ulcers and gangrene (see fig 1 ).

Three weeks before this event, he had presented to the A\&E department with bilateral painful thighs after routine and uneventful cardiac angiography. He was admitted on that occasion and treated with anticoagulant therapy. He was discharged home on the 10th day with warfarin.

His was known to have unstable angina, chronic obstructive airway disease, aortic aneurysm, and borderline hypercholesterolaemia, and he was a known ex-smoker. He was not known to be diabetic nor was his family history significant.

Physical examination revealed tender distal toes with intact distal pulses. Both his feet were
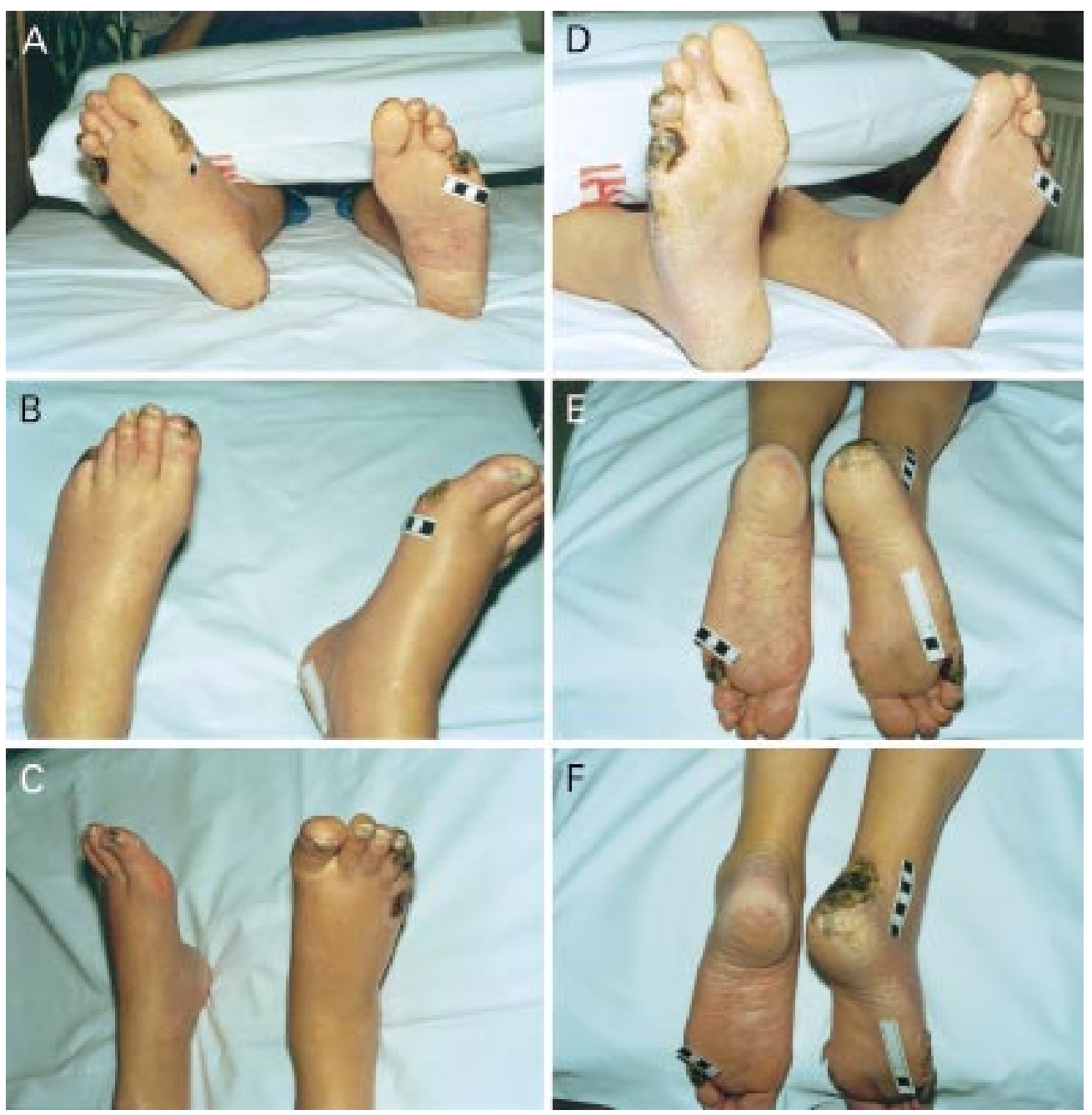

Figure 1 Patient's feet showing the progression from blisters to ulcers and gangrene $(A-F)$ (reproduced with patient's permission). warm and well perfused other than the gangrenous patches. Other neurovascular examinations of the lower limb yielded no abnormalities.

Laboratory investigation on admission revealed an eosinophilic count of $1.03 \times 10^{9} / 1$, erythrocyte sedimentation rate $34 \mathrm{~mm} /$ hour, urea $8.4 \mathrm{mmol} / 1$, and creatinine $138 \mu \mathrm{mol} / 1$. A dipstick test of his urine showed it to be positive for red blood cells. His creatine kinase was $1363 \mathrm{U} / 1$. His dorsalis pedis resting pressure indices were 0.99 and 0.97 for the left and right foot respectively.

\section{Questions}

(1) What is the clinical diagnosis? What could be the cause of the ulceration and gangrene in the feet?

(2) How can we confirm the diagnosis?

(3) What is the treatment and prognosis?
Department of Vascular Surgery, King George Hospital, Barley Lane, Goodmayes, Ilford, Essex IG3 8YB, UK A M Khan S Jacobs

Correspondence to: Mr Khan

dramirkhan@hotmail.com

Submitted 29 August 2000 Accepted 17 October 2000 


\section{Breathlessness after percutaneous biliary drainage}

\author{
H Patel, K S Hindle, G Tsavellas, A Huang
}

Answers on $p 795$.

Department of Academic Surgery, Chelsea and

Westminster Hospital, London SW10 9NH, UK

H Patel

G Tsavellas

A Huang

Wycombe Hospital, High Wycombe, Buckinghamshire K S Hindle

Correspondence to: Mr Huang

a.huang@ic.ac.uk

Submitted 14 August 2000 Accepted 17 October 2000

Answers on $p 795$.

Renal Unit, Glasgow Royal Infirmary, Glasgow, Scotland

N Joss

K Simpson

Correspondence to: Dr Nicola Joss, Renal Unit, Glasgow Royal Infirmary, 84 Castle Street, Glasgow G4 OSF, UK

njoss@compuserve.com

Submitted 22 March 2000 Accepted 6 November 2000 progressive jaundice and weight loss. She also had generalised pruritus and was passing dark urine and pale stools. On examination there was a palpable right upper quadrant abdominal mass. Plasma bilirubin level was $56 \mu \mathrm{mol} / 1$ and alkaline phosphatase was $350 \mathrm{U} / 1$. Serum amylase was within normal limits. Abdominal ultrasound scan showed a dilated biliary tree

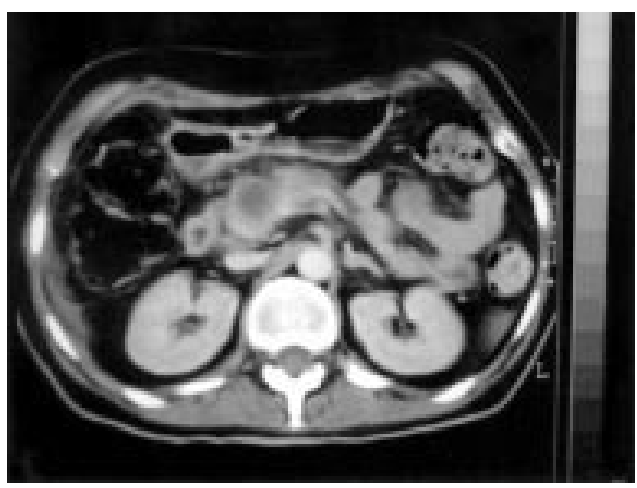

Figure 1 Abdominal computed tomogram.

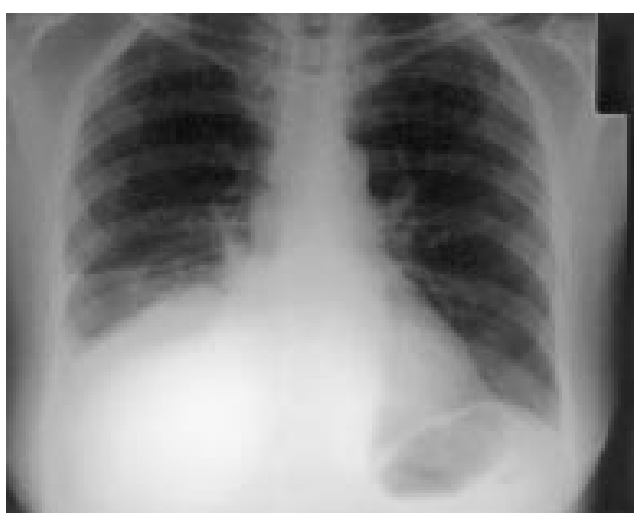

Figure 2 Thoracic radiograph.
A 77 year old woman presented with painless

\author{
N Joss, K Simpson
}

A 37 year old women presented with a 10 year history of episodes of weakness and palpitations. On one or two occasions each year she was admitted to her local hospital where her serum potassium concentration was always below the reference range with the lowest concentration reported at $2.2 \mathrm{mmol} / 1$ (3.5-5.0 $\mathrm{mmol} / \mathrm{l})$. The episodes were terminated with intravenous potassium. At other times she had mild weakness on exertion and occasional palpitations. These symptoms disappeared with oral potassium supplements. She was always normotensive. She had no diarrhoea or vomiting, her drug history consisted of oral amiloride

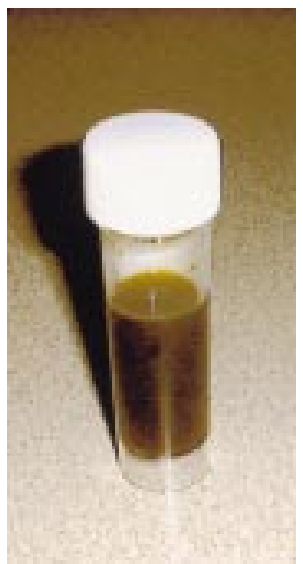

Figure 3 Pleural aspirate.

and a distended gallbladder but there was no evidence of gallstones. Abdominal computed tomography was performed (fig 1). The patient underwent an endoscopic retrograde cholangiopancreaticogram (ERCP) and insertion of a biliary stent, with resolution of the jaundice.

Ten days later the patient became jaundiced again and an abdominal ultrasound suggested stent occlusion with recurrent biliary tree dilatation. Percutaneous transhepatic cholangiography (PTC) with external biliary drainage was performed. The patient gradually became short of breath with right sided pleuritic pain and a thoracic radiograph was obtained (fig 2). Thoracocentesis was performed and the pleural aspirate is shown (fig 3 ).

\section{Questions}

(1) What is the most likely cause of her jaundice and why was this suspected clinically?

(2) What complication of PTC has occurred?

(3) What is the treatment of this complication?

\section{Episodic weakness in a young woman}

$15 \mathrm{mg} /$ day and a variable amount of effervescent potassium chloride supplements. She had a sister who had one minor episode of weakness but had not been investigated. She was referred to a renal unit for further investigations and to obtain a diagnosis.

On examination she looked well, with a blood pressure of $115 / 70 \mathrm{~mm} \mathrm{Hg}$, she was clinically euvolaemic, she had no signs of self induced vomiting, and she was not cushingoid.

Her initial investigations revealed the following serum electrolyte concentrations: sodium $137 \mathrm{mmol} / 1$ (135-145), potassium $3.0 \mathrm{mmol} / 1$ (3.5-5.0), chloride $100 \mathrm{mmol} / \mathrm{l}$ (97-107), 